\title{
Substrate-Embedded and Flip-Chip-Bonded Photodetector Polymer-Based Optical Interconnects: Analysis, Design, and Performance
}

\author{
Elias N. Glytsis, Senior Member, IEEE, Fellow, OSA, Nan M. Jokerst, Fellow, IEEE, Fellow, OSA, \\ Ricardo A. Villalaz, Student Member, IEEE, Student Member, OSA, Sang-Yeon Cho, Student Member, IEEE, \\ Shun-Der Wu, Student Member, IEEE, Student Member, OSA, Zhaoran Huang, Student Member, IEEE, \\ Martin A. Brooke, Member, IEEE, and Thomas K. Gaylord, Fellow, IEEE, Fellow, OSA
}

\begin{abstract}
The performance of three optoelectronic structures incorporating substrate-embedded InP-based inverted metal-semiconductor-metal photodetectors and/or volume holographic gratings are analyzed and compared at the primary optical communication wavelengths. These structures, in conjunction with optical-quality polymer layers, can be easily integrated into silicon microelectronic substrates for the purpose of implementing potentially low-cost high-data-rate chip-level or substrate-level optical interconnects. The structures are as follows: a) an evanescent-coupling architecture with a substrate-embedded photodetector, b) a volume-holographic-grating coupler architecture with a substrate-embedded photodetector, and c) a volume-holographic-grating coupler architecture with a flip-chip-bonded photodetector. It is found that the primary characteristic of the evanescent coupling architectures is the efficient performance for both TE and TM polarizations with the disadvantage of exponentially decreasing efficiency with increasing separation between the waveguide film layer and the photodetector layer. On the other hand, the primary characteristic of the volume holographic grating architectures is the possibility of wavelength and polarization selectivity and their independence on the separation between the photodetector layer and the waveguide. Comparison of the analysis with experimental results is also included in the case of the evanescent coupling into a substrate-embedded photodetector.
\end{abstract}

Index Terms-Electromagnetic coupling, electromagnetic radiation, gratings, optical couplers, optical interconnections, packaging, photodetectors.

\section{INTRODUCTION}

A $\mathrm{S}$ electronic system aggregate data rates rise and the sizes decrease, conventional electrical interconnections face multiple challenges at the backplane, board, and module levels. High-performance electrical interconnection systems inevitably trade off power consumption, area, and signal integrity (jitter, delay, skew). Architectural and design approaches, as well as technological innovation at the physical layer level, can be used to improve interconnection performance. However, physical limitations will ultimately force technology changes

Manuscript received December 16, 2002; revised June 26, 2003. This work was supported in part by the State of Georgia Yamacraw Mission and in part by the National Science Foundation under Grant ERC-94-02723.

The authors are with the School of Electrical and Computer Engineering and Microelectronics Research Center, Georgia Institute of Technology, Atlanta, GA 30332 USA.

Digital Object Identifier 10.1109/JLT.2003.818178 at the physical layer if performance gains are to continue well into the future. If low loss, high-speed, low-power, compact optical interconnections could be implemented at the board and module levels with simple interfaces, this could offer the designer a high-performance interconnect option in a relatively inexpensive board technology.

A number of quantitative comparisons of interconnection performance have been published discussing electrical and optical interconnections [1]-[3]. A critical issue is how to integrate optical interconnections into an electrical interconnection system. Optical interconnect approaches include free-space interconnects with diffractive optical elements [4], silicon optical bench interconnects [5], and guided-wave interconnections, including substrate-guided-mode interconnects [6], fiber-optic waveguides [7], and integrated waveguides [8]. This paper focuses on waveguide optical interconnections that are integrated directly onto the electrical interconnection package media such as boards and modules.

Electrical boards, modules, and integrated circuits are essentially planar, and thus, an embedded planar waveguide optical interconnection scheme matches the topography of the electrical system. One basic classification of waveguide optical interconnections embedded in a board is whether or not the optical beam is turned perpendicular to the waveguiding plane for electrical-to-optical and optical-to-electrical conversion interfaces. Mirrors (that represent localized-type coupling) and gratings (that represent distributed-type coupling) can be used to turn the optical beam to/from the waveguide into optoelectronic emission/detection devices. However, mirrors have the disadvantage of alignment sensitivity due to their localized-type coupling. On the other hand, gratings represent a distributed-type coupling and therefore can be more alignment tolerant. Gratings could be either of volume-holographic type or of surface-relief type. Furthermore, to achieve preferential coupling (toward a desired direction), slanted gratings need to be utilized. The slant does not complicate the fabrication process of the volume holographic gratings. However, slanted surface-relief gratings are generally difficult to fabricate especially in large areas and in multiple locations. Alternatively, emitters and detectors can be embedded in the waveguide using heterogeneous integration or monomaterial substrate/device integration. For example, silicon metal-semiconductor-metal (MSM) photodetectors have been proposed for embedded implementation of optical interconnects 
using beam turning elements such as slanted surface-relief gratings and $45^{\circ}$ micromirrors [9]. However, silicon MSM photodetectors are not as efficient as the InP-material-based inverted MSM photodetectors. Another advantage of the InP-material-based photodetectors is that their carrier lifetime is generally shorter thus permitting higher data-rate implementations.

In this paper, potentially low-cost fully substrate-embedded or flip-chip bonded optical interconnect architectures are analyzed and compared at the main optical communication wavelengths $(1.3 \mu \mathrm{m}$ and $1.55 \mu \mathrm{m})$. The architectures involve a volume-holographic grating coupling for beam turning or/and an embedded thin-film inverted-MSM photodetector for evanescent coupling. The architectures can be integrated on silicon microelectronic substrates with InP-material-based thin-film photodetectors and optical quality polymer layers that can be used as waveguides and/or volume holographic gratings. Three structures are explored as follows: a) an evanescent-coupling architecture with a substrate-embedded photodetector, b) a volume-holographic-grating coupler architecture with a substrate-embedded photodetector, and c) a volume-holographic-grating coupler architecture with a flip-chip-bonded photodetector. The customized volume-holographic gratings are recorded in photosensitive polymers such as the DuPont OmniDex613 (HRF600X) and can be laminated on top of a waveguide. All architectures are analyzed, optimized, and compared. Their primary characteristics are investigated as well as their advantages and disadvantages. The analysis of the three proposed architectures is presented in Section II. The design and optimization of the architectures are presented in Section III. Some fabrication issues are summarized in Section IV. Some preliminary experimental results are presented in Section V. Finally, the summary and the main conclusions of this paper are given in Section VI.

\section{ANALYsis of Proposed Structures}

The basic waveguiding structure is composed of a single-mode slab waveguide, as shown to the left of the $x$-axis in Fig. 1(a)-(c). The waveguide film layer has an index of refraction $n_{f}$ and a thickness $t_{2}$. The cover and the substrate are considered semi-infinite regions with refractive indexes $n_{c}$ and $n_{s}$, respectively. In Fig. 1(a)-(c), three structures are shown: in (a), an evanescent-coupling architecture with a substrate-embedded photodetector, in (b), a holographic grating coupler architecture with a substrate-embedded photodetector, and in (c), a holographic grating coupler architecture with a flip-chip-bonded photodetector. The objective of all of these architectures is the coupling of guided light incident from the left to the photodetector (either substrate embedded or flip-chip bonded) that is represented by the semi-infinite layer of refractive index $n_{D}$. In general, the photodetector region has a finite thickness (thin-film photodetector) and a complex refractive index representing the absorption of the optical power. However, for simplicity in this analysis, the photodetector region is modeled by a semi-infinite region since any optical power that enters this region does not return to the waveguide due to absorption. As a result, the finite-thickness photodetector region of complex refractive index can be simplified and be

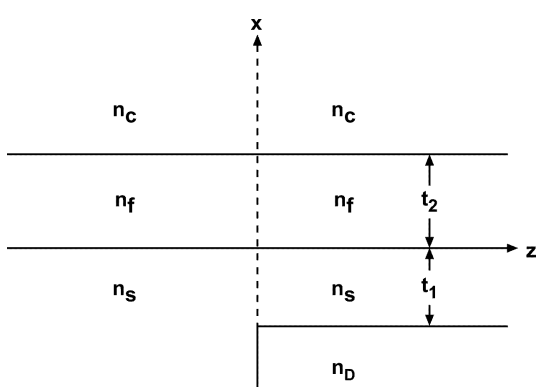

(a)

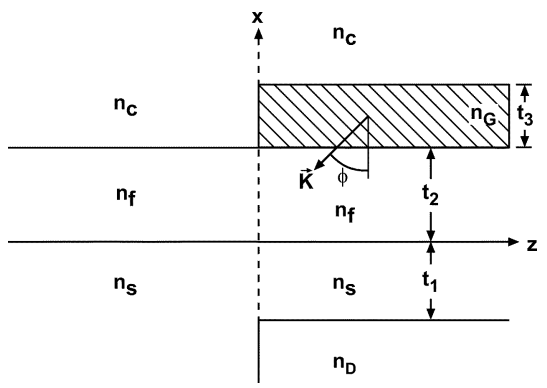

(b)

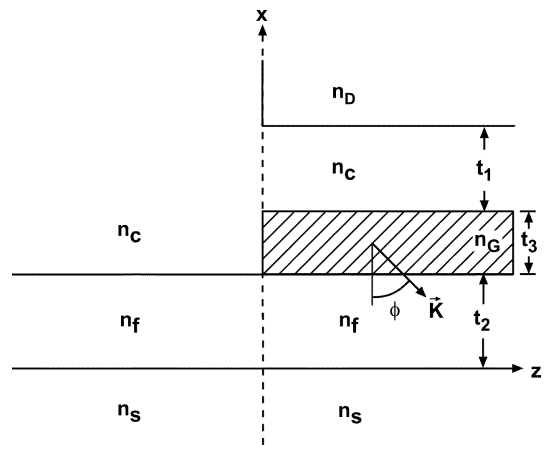

(c)

Fig. 1. (a) The geometry of evanescent coupling to the substrate-embedded photodetector. The single-mode optical waveguide has a thickness of $t_{2}$ and refractive indexes of $n_{c}, n_{f}$, and $n_{s}$ for the cover, film, and substrate layers, respectively. The embedded photodetector region starts at $z>0$, and the photodetector layer is at a distance $t_{1}$ from the film layer and has a refractive index $n_{D}>n_{f}>n_{s}>n_{c}$. (b) The geometry of holographic-grating coupling to the substrate-embedded photodetector. The single-mode waveguide and the embedded photodetector have the same parameters as in (a). The holographic grating extends from $z>0$ and has a thickness of $t_{3}$ and an average refractive index of $n_{G}$. The grating vector $\vec{K}$ and its slant angle $\phi$ are also shown. (c) The geometry of holographic-grating coupling to the flip-chip-bonded photodetector. All parameters are similar to those in (b).

represented by a semi-infinite region of real refractive index $n_{D}$. It is assumed that a single mode (TE or TM polarized) with optical power $P_{\text {inc }}$ is propagating in the slab waveguide from left to right. The theoretical models for the analysis and performance evaluation of all architectures are summarized next.

\section{A. Evanescent Coupling From Waveguide to Substrate-Embedded Photodetector}

The evanescent coupling architecture is shown in Fig. 1(a). The substrate-embedded photodetector is placed at a distance $t_{1}$ from the film layer of the waveguide (region of $z>0$ ). The refractive index of the photodetector ( $\operatorname{nn} P$-based material system) is assumed to be larger than any of the surrounding indexes, i.e., 
$n_{D}>n_{f}>n_{s}>n_{c}$. Therefore, when guided light enters the region of the embedded photodetector $(z>0)$, the evanescent tail (toward the substrate) of the guided mode "senses" the presence of the high index region of the photodetector because of the frustrated total internal reflection at the film-substrate boundary. As a result, and assuming that $t_{1}$ is on the order of the light wavelength, the optical power from the guided mode radiates into the embedded photodetector region, where it is absorbed and produces a photocurrent. Thus, the optical signal transferred by the optical waveguide mode is being converted into an electrical signal that is transmitted via the photodetector circuitry to the electronic components of the substrate.

The radiation (leakage) of the guided-mode power in region $z>0$ can be represented using a leaky-mode description of the electromagnetic field [10], [11]. Under the leaky-mode description, the electromagnetic field in the waveguide for $z>0$ can be manifested by a complex propagation constant $\tilde{\beta}=\beta-j \alpha$, and the optical field $U(x, z)$ (electric or magnetic) can be expressed as $U(x, z)=U(x) \exp (-j \tilde{\beta} z)=U(x) \exp (-j \beta z) \exp (-\alpha z)$, where $U(x)$ corresponds to the field profile along the $x$ direction. The optical power $P(z)$ that is radiated and absorbed into the embedded detector region can then be expressed as

$$
P(z)=(1-R) P_{\text {inc }}\left(1-e^{-2 \alpha z}\right)
$$

where $R$ represents the percentage of the reflected power of the incident mode when it enters the photodetector $(z>0)$ region. The reflected power $R P_{\text {inc }}$ depends on the separation $t_{1}$ between the film layer and the photodetector layer. It is expected that the smaller $t_{1}$ is, the stronger the radiation and, consequently, the absorption of the optical power in the photodetector would be. However, the smaller $t_{1}$ is, the stronger the discontinuity at the $z=0$ interface becomes, thus resulting in higher reflection losses of the incident mode (larger $R$ ). Therefore, there is an optimal separation $t_{1}$ between the film layer and the photodetector layer that will maximize the power $P(z=L)$ for a given detector layer length (along the $z$-axis) $L$. The imaginary part $\alpha$ of the complex propagation constant of the leaky mode can be defined as the radiation coupling coefficient since the leakage of the mode represents the power radiated into the photodetector area. The power that can radiate into the cover (air) area (for $z>0$ ) can be neglected since those leaky modes will not be strongly excited at the $z=0$ interface. The radiation coupling coefficient $\alpha$ can be calculated using the argument principle method (APM) [12], [13]. The latter method is a rigorous mathematical technique based on complex analysis [14] and is capable of finding the zeros (leaky-mode propagation constants $\tilde{\beta}=\beta-j \alpha$ ) of any analytic function (the waveguide dispersion relation) in the complex plane. Therefore, the radiation coupling coefficient $\alpha$ in (1) can be easily determined. However, the APM method cannot be used for the estimation of the percentage of the reflected power $R$. In order to calculate $R$, the finite-difference frequency-domain (FDFD) method is used [15]-[17] in conjunction with Bérenger's perfectly matched layer and absorbing boundary conditions [18]. With $\alpha$ and $R$ calculated using the APM and FDFD methods, respectively, the power absorbed in the detector area as well the optimal selection of $t_{1}$ can be determined.

\section{B. Holographic-Grating Coupling From Waveguide to Substrate-Embedded Photodetector}

The holographic-grating architecture for coupling from the waveguide to the substrate-embedded photodetector is shown in Fig. 1(b). The embedded photodetector is placed again at a distance $t_{1}$ from the film layer of the waveguide (region of $z>0$ ) and has similar parameters as in the evanescent coupling architecture. The holographic grating (usually composed of a photopolymer) is on the top of the waveguide for $z>0$. The average refractive index of the grating is $n_{G}$, while the grating vector is defined as $\vec{K}=K_{x} \hat{x}+K_{z} \hat{z}=-(2 \pi / \Lambda)(\hat{x} \cos \phi+\hat{z} \sin \phi)$, with $\phi$ being the slant angle $\left(0<\phi<90^{\circ}\right), \Lambda$ the period of the holographic grating, and $\hat{x}, \hat{z}$ corresponding to the unit vectors along the $x$ and $z$ directions, respectively. In order for the incident-from-the-left guided mode to remain guided in the $z>0$ region, it is assumed that $t_{1}$ is several wavelengths long so that the frustrated total internal reflection at the film-substrate boundary can be neglected and that $n_{G}<n_{f}$. Therefore, when guided light enters the region of the embedded photodetector $(z>0)$, the evanescent tail (in this case toward the cover) of the guided mode "senses" the presence of the periodic index modulation of the holographic grating region. As a result, optical power from the guided mode can get diffracted preferentially toward the embedded photodetector region, where it is absorbed and produces a photocurrent as in the evanescent coupling architecture of Fig. 1(a). Thus, in the holographic-grating coupling architecture, the optical signal transferred by the optical waveguide mode is being converted into an electrical signal that is transmitted via the photodetector circuitry to the electronic components of the substrate. The major difference relative to the evanescent-coupling architecture is that the grating could have wavelength- and polarization-sensitive (to the incident mode) performance in contrast to the evanescent-coupling architecture.

The holographic grating has a relative permittivity variation $\varepsilon_{G}$ expressed by

$$
\begin{aligned}
\varepsilon_{G}(x, z) & =n_{G}^{2}+\varepsilon_{1} \cos (\vec{K} \cdot \vec{r}) \\
& =n_{G}^{2}+\varepsilon_{1} \cos \left(K_{x} x+K_{z} z\right)
\end{aligned}
$$

where $\varepsilon_{1}$ is the grating modulation and $\vec{r}=x \hat{x}+y \hat{y}+z \hat{z}$. A higher number of harmonics can be easily added in the above expression without affecting the analysis presented below. However, for holographic gratings, a sinusoidally varying relative permittivity expresses reliably the grating modulation in most cases. When the guided mode enters the region of the embedded photodetector $(z>0)$, electromagnetic power diffracts toward the photodetector region where it is absorbed. The diffraction (leakage) of the power of the mode in the region with $z>0$ can be represented again by a leaky-mode description of the electromagnetic field in this region [19]-[24]. Under the leaky-mode description, the electromagnetic field in the $z>0$ region can be represented by the complex propagation constant $\tilde{\beta}=\beta-j \alpha$ 
and the optical field in each layer (electric or magnetic for TE or TM polarization respectively) can be expressed according to the rigorous coupled-wave analysis [25], [26] as

$$
\begin{aligned}
U_{m}(x, z)= & \hat{y} \sum_{i} S_{m i}(x) \exp (j i \vec{K} \cdot \vec{r}) \exp (-j \tilde{\beta} z) \\
= & \hat{y} \sum_{i} S_{m i}(x) \exp \left[j i\left(K_{x} x+K_{z} z\right)\right] \\
& \times \exp (-j \beta z) \exp (-\alpha z)
\end{aligned}
$$

where $m$ corresponds to the grating layer (of thickness $t_{3}$ ), to the waveguide film layer (of thickness $t_{2}$ ), or to the substrate layer (of thickness $t_{1}$ ); $S_{m i}$ are the spatial harmonics in each layer; and $i$ corresponds to the number of the diffracted orders that are retained in the analysis. The fields in the cover and photodetector regions can be expressed as

$$
\begin{aligned}
U_{c}(x, z) & =\hat{y} \sum_{i} R_{i} \exp \left(-j \vec{k}_{c i} \cdot \vec{r}\right) \\
U_{D}(x, z) & =\hat{y} \sum_{i} T_{i} \exp \left(-j \vec{k}_{D i} \cdot \vec{r}\right)
\end{aligned}
$$

where $R_{i}$ and $T_{i}$ are the amplitudes of the diffracted waves in the cover and photodetector regions, respectively. The wavevectors $\vec{k}_{c i}=\left(\tilde{\beta}-i K_{z}\right) \hat{z}+k_{c x i} \hat{x}$ and $\vec{k}_{D i}=\left(\tilde{\beta}-i K_{z}\right) \hat{z}+k_{D x i} \hat{x}$ correspond to the wavevectors for each diffracted order $i$ in the cover and photodetector regions, respectively. The $x$-components of the wavevectors $k_{c x i}$ and $k_{D x i}$ can be determined from the plane-wave dispersion relations $k_{\ell x i}^{2}+k_{\ell z i}^{2}=k_{0}^{2} n_{\ell}^{2}$ (with $\ell=c$ or $D$ ). Furthermore, due to the complex propagation constant $\tilde{\beta}$, the $x$ components $k_{c x i}$ and $k_{D x i}$ of the diffracted wavevectors have to be selected appropriately [23], [24], [27] in order to correspond to physical radiation directions of the diffracted fields. Using the electric and magnetic field components of the optical waves in the various regions in conjunction with the electromagnetic boundary conditions and taking into account the rigorous coupled-wave analysis solution method, the complex propagation constant $\tilde{\beta}$ can be determined as a solution of a transcendental equation $\operatorname{det}[\mathbf{M}(\tilde{\beta})]=0$, where $\mathbf{M}$ is a matrix of size $8 N \times 8 N$ and $N$ is the number of diffracted orders retained in the analysis.

After the calculation of $\tilde{\beta}$, the optical power $P(z)$ that is diffracted and absorbed in the embedded detector region can then be expressed by

$$
P(z)=(1-R) \eta_{D} P_{\text {inc }}\left(1-e^{-2 \alpha z}\right)
$$

where $R$ again represents the percentage of the reflected power of the incident mode when it enters the photodetector $(z>0)$ region and $\eta_{D}$ is the preferential coupling ratio [22]-[24] that is defined as the fraction of the total power that is diffracted into the photodetector region. As in the evanescent coupling architecture, the reflected power $R P_{\text {inc }}$ depends strongly (exponentially) on the separation $t_{1}$ between the film layer and the photodetector layer. However, in this case $t_{1}$ should be selected several wavelengths in length; otherwise (as will be shown in Section III) the evanescent coupling prevails as compared to the holographic-grating coupling. Therefore, with this selection of $t_{1}$, the effect of the reflection at $z=0$ becomes small since the power reflected would depend only on the presence of the grating layer, which has a much smaller refractive index mismatch. With $\alpha$ and $\eta_{D}$ calculated using the rigorous coupled-wave analysis, and $R$ using the FDFD method, the power absorbed in the detector area can be calculated as a function of the coupler length and can also be compared to the power coupled using the evanescent coupling architecture.

\section{Holographic-Grating Coupling From Waveguide to Flip-Chip-Bonded Photodetector}

The holographic-grating architecture for coupling from the waveguide to the flip-chip-bonded photodetector is shown in Fig. 1(c). In this architecture, the photodetector is placed at a distance $t_{1}$, which is large in comparison to the optical wavelength due to the flip-chip bonding bumps (on the order of $50 \mu \mathrm{m}$ ). Therefore, no evanescent coupling between the waveguide and the detector layers exists. The coupling in this case is solely provided by the grating, which in this case is designed to diffract power in the positive $x$-direction. The grating vector in this case is defined as (in order to have $0<\phi<90^{\circ}$ as before) $\vec{K}=K_{x} \hat{x}+K_{z} \hat{z}=(2 \pi / \Lambda)(-\hat{x} \cos \phi+\hat{z} \sin \phi)$, with $\phi$ being the slant angle and $\Lambda$ the period of the holographic grating as before. The analysis of this architecture is very similar to that of grating coupling to the substrate-embedded photodetector presented in the previous section. The only difference is that (3) now holds for the grating region (of thickness $t_{3}$ ), the cover region (of thickness $t_{1}$ ), and the film region (of thickness $t_{2}$ ). In addition, the fields in photodetector and substrate regions can be expressed as

$$
\begin{aligned}
U_{D}(x, z) & =\hat{y} \sum_{i} R_{i} \exp \left(-j \vec{k}_{D i} \cdot \vec{r}\right) \\
U_{s}(x, z) & =\hat{y} \sum_{i} T_{i} \exp \left(-j \vec{k}_{s i} \cdot \vec{r}\right)
\end{aligned}
$$

where $R_{i}$ and $T_{i}$ are the amplitudes of the diffracted waves in the photodetector and substrate regions, respectively. The wavevectors $\vec{k}_{D i}=\left(\tilde{\beta}-i K_{z}\right) \hat{z}+k_{D x i} \hat{x}$ and $\vec{k}_{s i}=\left(\tilde{\beta}-i K_{z}\right) \hat{z}+k_{s x i} \hat{x}$ correspond to the wavevectors for each diffracted order $i$ in the photodetector and substrate regions, respectively. The $k_{D x i}$ and $k_{s x i}$ components can be determined from the plane-wave dispersion relations similarly to the procedure described in the previous section. The rigorous coupled-wave analysis in conjunction with the leaky-mode field description is used again for the determination of the power that is coupled into the photodetector region. This power can be expressed again by (6).

\section{Design and Optimization of Coupling Structures}

The parameters used for the implementation of the architectures shown in Fig. 1(a)-(c) are based on polymer materials and for the primary telecommunications wavelengths, i.e., $\lambda_{0}=1.3$ and $1.55 \mu \mathrm{m}$. For example, for the substrate-embedded-photodetector architectures [Fig. 1(a) and (b)], the film layer is composed of Ultem polymer [28] of refractive index $n_{f}=1.65$. In the latter cases, the substrate material is comprised of benzocyclobutane (BCB) polymer [29] of refractive index $n_{s}=1.55$. In the case of the flip-chip-bonded-photodetector architecture [Fig. 1(c)], the waveguide material is BCB of $n_{f}=1.55$ and the substrate material is $\mathrm{SiO}_{2}$ of $n_{s}=1.45$. The photodetector layer is composed of a InP-based material and has an average 
refractive index $n_{D}=3.7$. The cover area is assumed to be air $\left(n_{c}=1.0\right)$. The holographic grating is recorded in the DuPont photopolymer OmniDex613 (HRF600X) of a refractive index $n_{G}=1.50$. The photopolymer is available in laminating sheets of thickness $t_{3}=10 \mu \mathrm{m}$ after the removal of the Mylar protective layers. Therefore, the thickness of the photopolymer is always taken to be $10 \mu \mathrm{m}$. For all grating designs, it was assumed that the refractive index modulation is $\varepsilon_{1}=2 n_{G} \Delta n$ with $\Delta n=0.02$, which corresponds to the photopolymer used. The waveguide is designed to be single-mode for both wavelengths of interest. The separation $t_{1}$ between film and photodetector layers varies depending on the architecture under investigation.

\section{A. Evanescent Coupling of Waveguide to Substrate-Embedded Photodetector}

In the case of the evanescent coupling architecture, it is very important to determine the optimal separation of film and photodetector layers in order to maximize the power that can be absorbed [in (1), both $\alpha$ and $R$ depend on $t_{1}$ ]. For this reason, the percentage of the reflected power $P_{\text {refl }}=R$ needs to be determined as a function of $t_{1}$ for both wavelengths of interest $\left(\lambda_{0}=1.3\right.$ and $\left.1.55 \mu \mathrm{m}\right)$ and for both incident polarizations (TE or TM incident mode). As was discussed in previous sections, the FDFD method was employed for this calculation. The waveguide film layer thickness was selected to be $t_{2}=1.0 \mu \mathrm{m}$ in this case. For the FDFD numerical implementation, the structure was enclosed around the $z=0$ interface in a computational box [17] of size $2 \mu \mathrm{m}$ along the $z$-direction and 13-15 $\mu \mathrm{m}$ along the $x$-direction including an absorbing boundary layer 2-3 $\mu \mathrm{m}$ thick. The grid point separation was $\lambda_{0} / 20 n_{\max }$ or smaller where $n_{\max }$ is the maximum refractive index (in this case $n_{\max }=n_{D}=3.7$ ). The incident mode was the $\mathrm{TE}_{0}$ or the $\mathrm{TM}_{0}$ of the single-mode waveguide. The FDFD method gives the complete electromagnetic field description. For example, in Fig. 2(a) and (b), the electric field intensity $\left(|U|^{2}=\left|E_{y}\right|^{2}\right)$ and the magnetic field intensity $\left(|U|^{2}=\left|H_{y}\right|^{2}\right)$ are shown for $\mathrm{TE}_{0}$ and $\mathrm{TM}_{0}$ incident modes, respectively, for $t_{1}=0 \mu \mathrm{m}$ and $\lambda_{0}=1.3 \mu \mathrm{m}$. In these figures the absorbing regions of the computational box are not shown since these regions do not correspond to the real structure but are used merely to absorb the power radiating away from the structure [17]. The dark regions represent areas of high field (electric or magnetic) intensity $\left(\left|E_{y}\right|^{2}\right.$ or $\left.\left|H_{y}\right|^{2}\right)$. The boundaries between various regions and the refractive indexes are also shown to facilitate understanding. The presence of the high index photodetector layer causes reflection losses at the $z=0$ plane. The interference maxima and minima can be seen in the waveguide film layer for both cases, as well as the radiation into the photodetector layer. Using discrete Fourier transforms of the electromagnetic fields [17], it is possible to determine the percentage of the reflected power as a function of $t_{1}$. The percentage of the reflected power $P_{\text {refl }}=R$ is shown in Fig. 3 as function of $t_{1}$ for both $\mathrm{TE}_{0}$ and $\mathrm{TM}_{0}$ incident guided modes and for both $\lambda_{0}=1.3 \mu \mathrm{m}$ and $\lambda_{0}=1.55 \mu \mathrm{m}$ free-space wavelengths. From this figure it can be seen that the reflected power depends exponentially on $t_{1}$ and is negligible for detector-waveguide separations $t_{1}>2 \mu \mathrm{m}$.

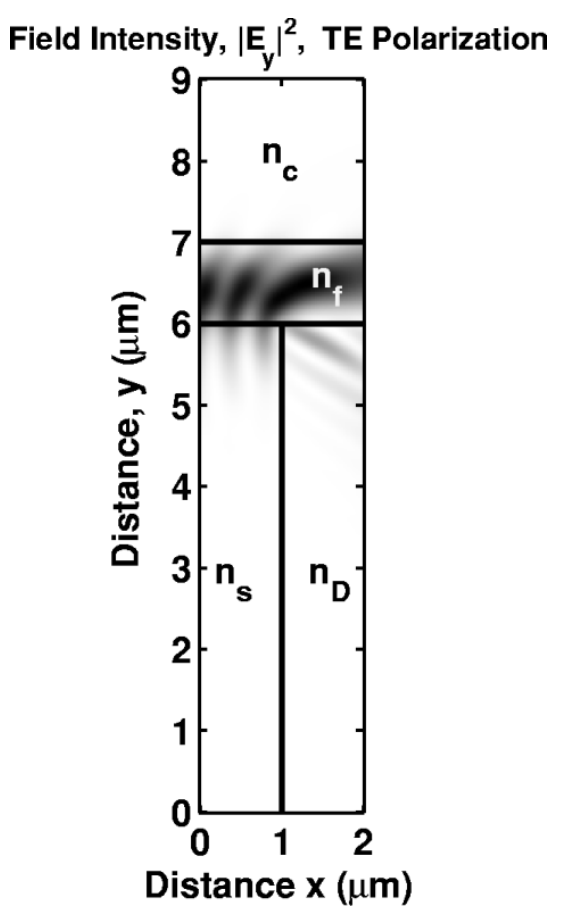

(a)

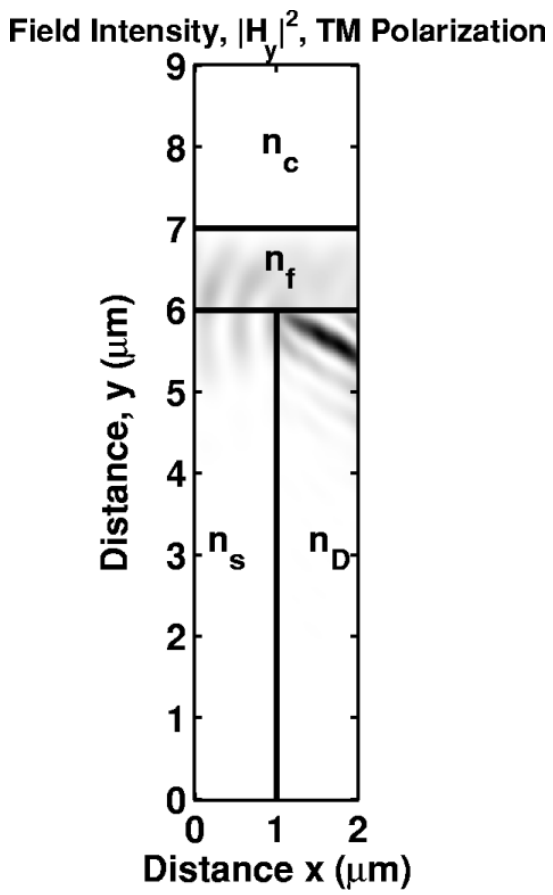

(b)

Fig. 2. (a) The electric field intensity pattern $\left|E_{y}\right|^{2}$ for a $\mathrm{TE}_{0}$ incident mode. The mode is propagating from left to right and is incident on the $z=0$ waveguide discontinuity (due to the presence of the photodetector layer, with $\left.t_{1}=0\right)$. The dark regions represent areas of high electric field intensity. The boundaries between various regions and the refractive indexes are also shown. The free-space wavelength of the light is $1.3 \mu \mathrm{m}$. This is a special case of Fig. 1(a) for $t_{1}=0$. (b) Same as in (a) but the magnetic field intensity $\left|H_{y}\right|^{2}$ is shown for a $\mathrm{TM}_{0}$ incident mode.

Using the APM method [12], the radiation coupling coefficient $\alpha$ can also be determined. This is shown in Fig. 4 as a function of the separation distance $t_{1}$ (between waveguide and photodetector) for both $\mathrm{TE}_{0}$ and $\mathrm{TM}_{0}$ incident polarizations and for both $\lambda_{0}=1.3 \mu \mathrm{m}$ and $\lambda_{0}=1.55 \mu \mathrm{m}$ free-space wavelengths. 


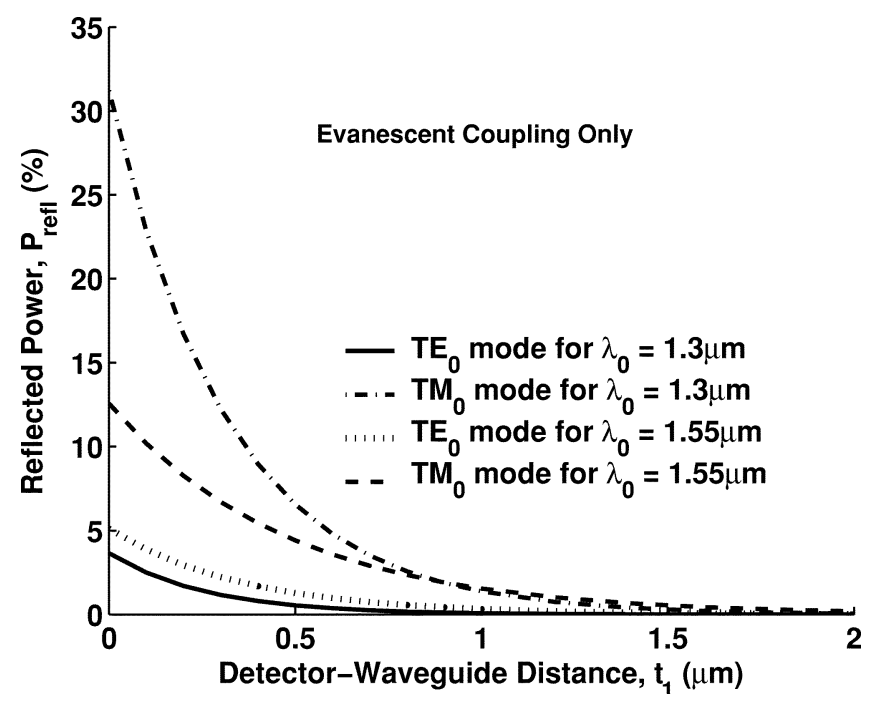

Fig. 3. Percentage of reflected power $P_{\text {refl }}=R$ at the $z=0$ waveguide discontinuity as a function of detector-waveguide (film) distance $t_{1}$ for the case of the evanescent coupling architecture. The various line types show the normalized reflected power $P_{\text {refl }}$ for both $\mathrm{TE}_{0}$ and $\mathrm{TM}_{0}$ incident modes as well as for $\lambda_{0}=1.3$ and $1.55 \mu \mathrm{m}$ free-space wavelengths.

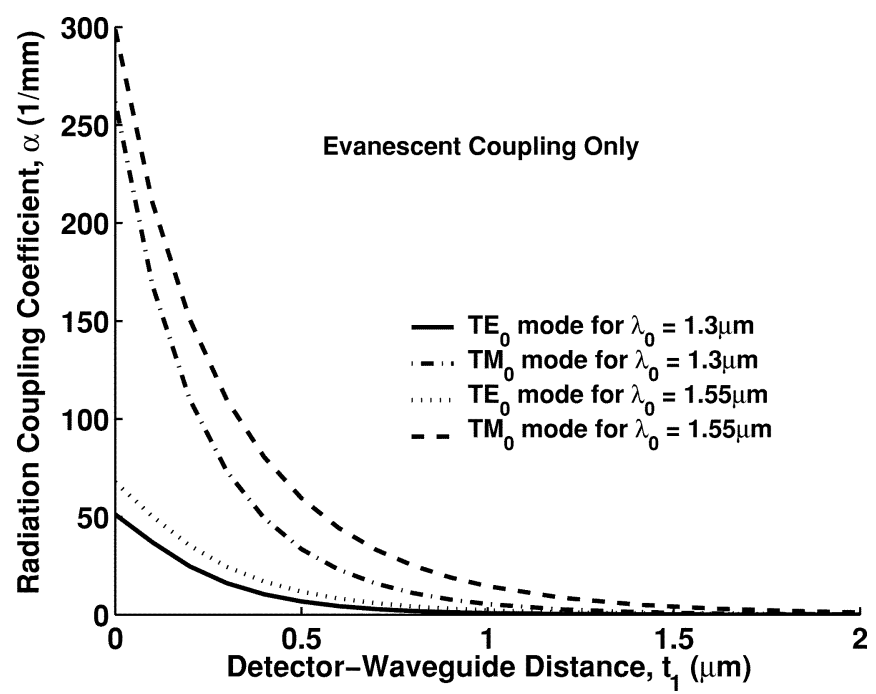

Fig. 4. Radiation coupling coefficient $\alpha$ for the leaky waveguide formed in the region $z>0$ as a function of detector-waveguide (film) distance $t_{1}$ for the case of the evanescent coupling architecture. The various line types show the radiation coupling coefficient $\alpha$ for both $\mathrm{TE}_{0}$ and $\mathrm{TM}_{0}$ incident modes as well as for $\lambda_{0}=1.3$ and $1.55 \mu \mathrm{m}$ free-space wavelengths.

From Fig. 4, it can also be seen that the radiation coupling coefficient reduces exponentially as the separation distance between detector and waveguide increases. This means that the presence of the photodetector layer will not cause the guided light to radiate ("leak") into the photodetector layer for large $t_{1}$.

The total normalized power that is coupled into the photodetector layer $P_{\text {det }} / P_{\text {inc }}=P(z=L) / P_{\text {inc }}$, where $P(z)$ is given by (1) and $L$ is the length (along the $z$-direction) of the photodetector, is shown in Fig. 5(a) and (b) as a function of the separation $t_{1}$ for $L=100$ and $250 \mu \mathrm{m}$, respectively, for both $\mathrm{TE}_{0}$ and $\mathrm{TM}_{0}$ incident polarizations and for both $\lambda_{0}=1.3$ and $1.55 \mu \mathrm{m}$ free-space wavelengths. From these figures, it can be seen that

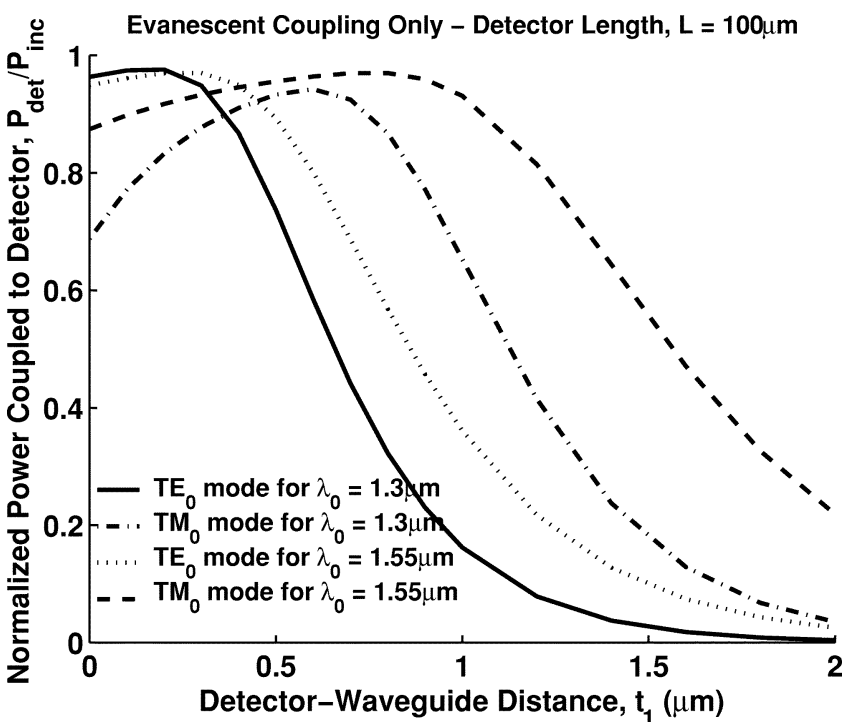

(a)

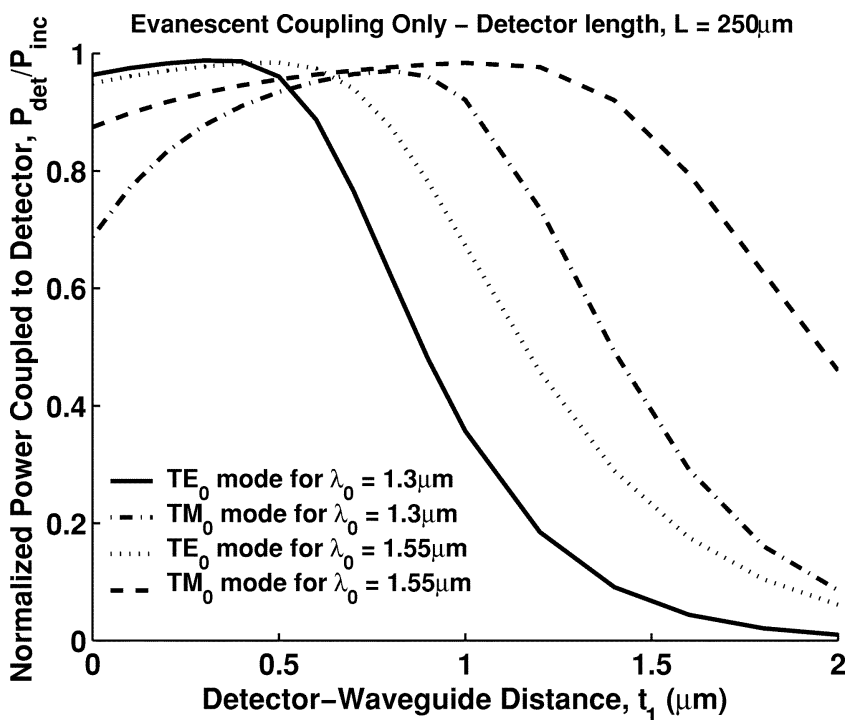

(b)

Fig. 5. Normalized power coupled to the detector $P_{\text {det }} / P_{\text {inc }}=P(z=$ $L) / P_{\text {inc }}$ for the leaky waveguide formed in the region $z>0$ as a function of detector-waveguide (film) distance $t_{1}$ for the case of the evanescent coupling architecture. The various line types show the normalized power $P_{\text {det }} / P_{\text {inc }}$ for both $\mathrm{TE}_{0}$ and $\mathrm{TM}_{0}$ incident modes as well as for $\lambda_{0}=1.3$ and $1.55 \mu \mathrm{m}$ free-space wavelengths. (a) For detector length $L=100 \mu \mathrm{m}$ and (b) for detector length $L=250 \mu \mathrm{m}$.

TABLE I

Optimal SeParation $t_{1}$ (IN $\mu \mathrm{m}$ ) Between WaVeguide Film Layer AND PHOTODETECTOR LAYER IN THE EVANESCENT COUPLING ARCHITECTURE FOR BOTH TE AND TM $_{0}$ INCIDENT MODES AND FOR $\lambda_{0}=1.3 \mu \mathrm{m}$ AND $1.5 \mu \mathrm{m}$ FREE-SPACE WAVELENGTHS AND $L=100$ AND $250 \mu \mathrm{m}$. AlL $t_{1}$ SEPARATIONS ARE ROUNDED TO A TENTH OF A MiCROMETER

\begin{tabular}{ccccc}
\hline \hline & \multicolumn{2}{c}{$L=100 \mu \mathrm{m}$} & \multicolumn{2}{c}{$L=250 \mu \mathrm{m}$} \\
\hline & $\lambda_{0}=1.3 \mu \mathrm{m}$ & $\lambda_{0}=1.55 \mu \mathrm{m}$ & $\lambda_{0}=1.3 \mu \mathrm{m}$ & $\lambda_{0}=1.55 \mu \mathrm{m}$ \\
\hline \hline$T E_{0}$ & 0.3 & 0.4 & 0.4 & 0.5 \\
$T M_{0}$ & 0.6 & 0.8 & 0.9 & 1.0 \\
\hline \hline
\end{tabular}

there is an optimal separation $t_{1}$ for a given detector length, incident mode polarization, and wavelength. The optimal separations are summarized in Table I for the abovementioned two 


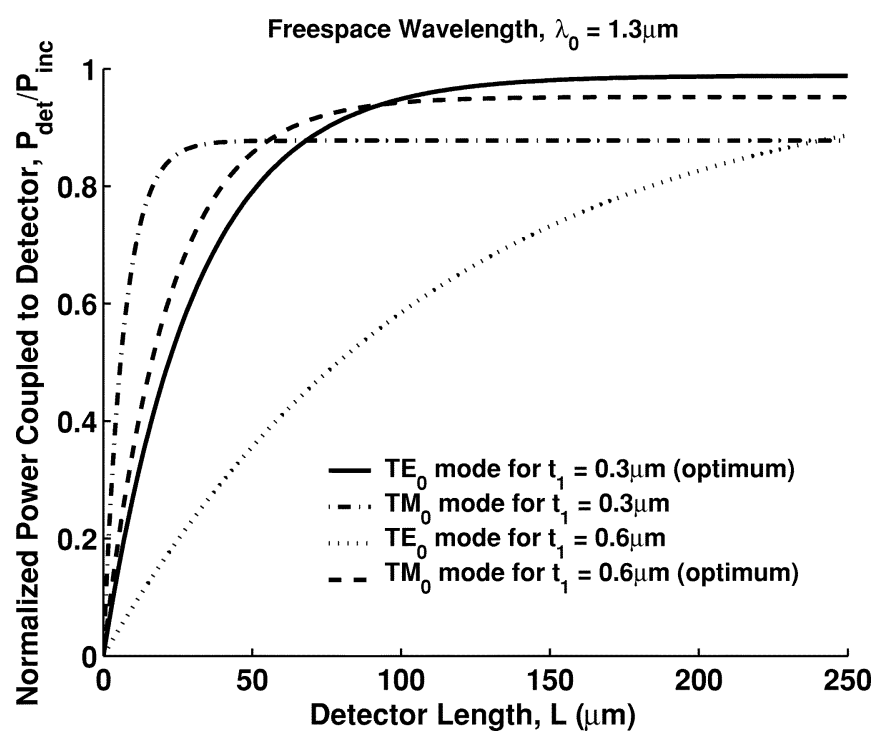

(a)

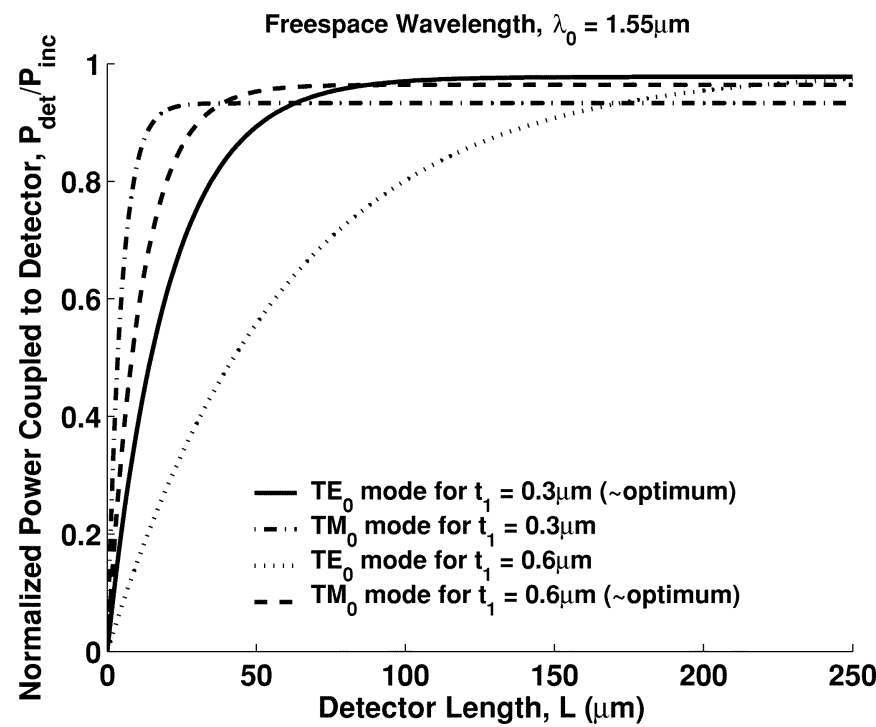

(b)

Fig. 6. Normalized power coupled to the detector $P_{\text {det }} / P_{\text {inc }}=P(z=$ $L) / P_{\text {inc }}$ for the leaky waveguide formed in the region $z>0$ as a function of the detector length for the case of the evanescent coupling architecture. The various line types show the normalized power $P_{\text {det }} / P_{\text {inc }}$ for both $\mathrm{TE}_{0}$ and $\mathrm{TM}_{0}$ incident modes as well as for optimal, near optimal, and nonoptimal $t_{1}$ separation. (a) For free-space wavelength $\lambda_{0}=1.3 \mu \mathrm{m}$. For this wavelength the optimal $t_{1}$ values from Table $\mathrm{I}$ are for the $\mathrm{TE}_{0}$ mode $t_{1}=0.3 \mu \mathrm{m}$ and for the $\mathrm{TM}_{0}$ mode $t_{1}=0.60 \mu \mathrm{m}$. (b) For free-space wavelength $\lambda_{0}=1.55 \mu \mathrm{m}$ using the optimal separations $t_{1}$ specified for the $\lambda_{0}=1.3 \mu \mathrm{m}$ free-space wavelength.

detector lengths of 100 and $250 \mu \mathrm{m}$. The optimal $t_{1}$ values are rounded to a tenth of a micrometer for realistic fabrication as well as to account for the relatively flat peaks of the curves shown in Fig. 5(a) and (b).

The total normalized power that is coupled into the photodetector layer $P_{\text {det }} / P_{\text {inc }}=P(z=L) / P_{\text {inc }}$ is also shown in Figs. 6 and 7 as a function of the detector length for optimal or nonoptimal $t_{1}$ separations for both $\mathrm{TE}_{0}$ and $\mathrm{TM}_{0}$ incident modes and for both $\lambda_{0}=1.3$ and $1.55 \mu \mathrm{m}$ free-space wavelengths. Specifically, in Fig. 6(a), $t_{1}$ is selected to be either $0.3 \mu \mathrm{m}$ (optimal value for $\mathrm{TE}_{0}$ mode and $\lambda_{0}=1.3$ ) or $0.6 \mu \mathrm{m}$ (optimal value for $\mathrm{TM}_{0}$ mode and $\lambda_{0}=1.3 \mu \mathrm{m}$ ). Fig. 6(b) shows

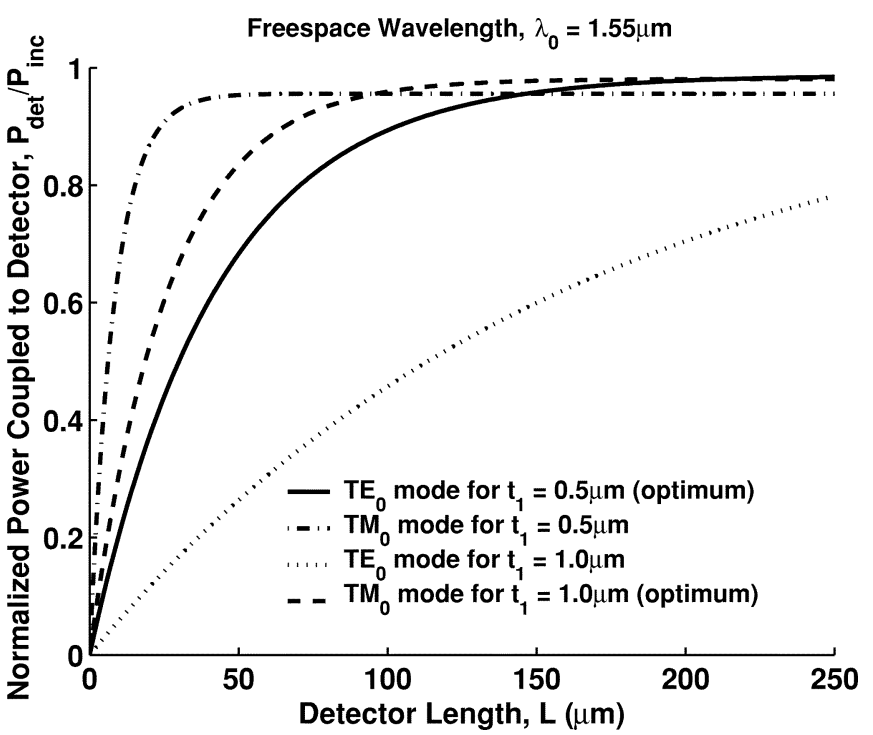

(a)

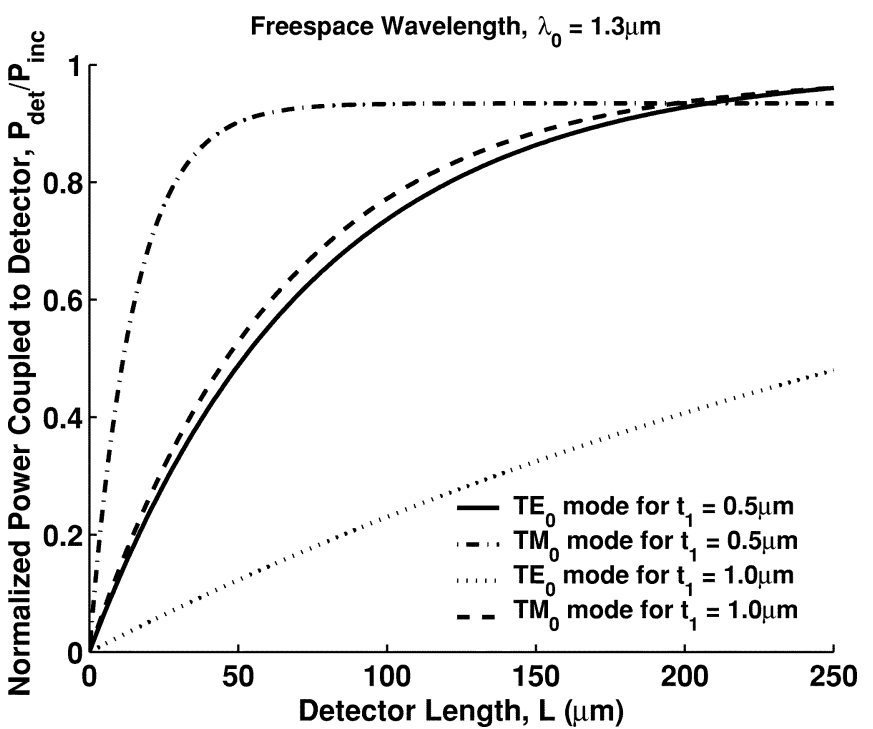

(b)

Fig. 7. Normalized power coupled to the detector $P_{\text {det }} / P_{\text {inc }}=P(z=$ $L) / P_{\text {inc }}$ for the leaky waveguide formed in the region $z>0$ as a function of the detector length for the case of the evanescent coupling architecture. The various line types show the normalized power $P_{\text {det }} / P_{\text {inc }}$ for both $\mathrm{TE}_{0}$ and $\mathrm{TM}_{0}$ incident modes as well as for optimal, near optimal, and nonoptimal $t_{1}$ separation. (a) For free-space wavelength $\lambda_{0}=1.55 \mu \mathrm{m}$. For this wavelength the optimal $t_{1}$ values from Table $\mathrm{I}$ are as follows: for the $\mathrm{TE}_{0}$ mode $t_{1}=0.5 \mu \mathrm{m}$ and for the $\mathrm{TM}_{0}$ mode $t_{1}=1.0 \mu \mathrm{m}$. (b) For free-space wavelength $\lambda_{0}=1.3 \mu \mathrm{m}$ using the optimal separations $t_{1}$ specified for the $\lambda_{0}=1.55 \mu \mathrm{m}$ free-space wavelength.

the performance of the architecture for $\lambda_{0}=1.55 \mu \mathrm{m}$ and for $t_{1}$ separations, which are optimal for the $\lambda_{0}=1.3 \mu \mathrm{m}$ free-space wavelength. Similarly, in Fig. 7(a), $t_{1}$ is selected to be either $0.5 \mu \mathrm{m}$ (optimal value for $\mathrm{TE}_{0}$ mode and $\lambda_{0}=1.55$ ) or $1.0 \mu \mathrm{m}$ (optimal value for $\mathrm{TM}_{0}$ mode and $\lambda_{0}=1.55 \mu \mathrm{m}$ ). Fig. 7(b) shows the performance of the architecture for $\lambda_{0}=1.3 \mu \mathrm{m}$ and for $t_{1}$ separations, which are optimal for the $\lambda_{0}=1.55 \mu \mathrm{m}$ free-space wavelength. From Figs. 6 and 7, it is deduced that 
there is a possible selection of $t_{1}\left(t_{1}=0.3 \mu \mathrm{m}\right)$ that makes the evanescent coupling efficient for both wavelengths and polarizations. In summary, the selection of the $t_{1}$ separation depends on the incident mode, the detector length, and the wavelength of operation. In addition, it can be seen that the percentage of the incident power that can be absorbed in the photodetector area can be as high as $~ 98 \%$.

\section{B. Holographic-Grating Coupling From Waveguide to Substrate-Embedded Photodetector}

In the case of the holographic-grating coupling architecture, the design of the grating is very important. The grating is designed in such a way that the first-order diffracted toward the photodetector region could be either normal or at a specified angle to the layer interfaces. Furthermore, the slanted grating configuration guarantees that a negligible amount of light will be diffracted into the cover direction due to its preferential diffraction properties. The grating design is based on the phase-matching conditions for the first diffracted order [23], [24]. If the desired order along the $-x$ direction forms an angle $\theta_{s}$ with respect to the $-x$-axis, then the grating vector components $K_{x}$ and $K_{z}$ are given by

$$
\begin{aligned}
\beta-K_{z} & =k_{0} n_{s} \sin \theta_{s} \\
\left(\beta-K_{z}\right)^{2}+K_{x}^{2} & =k_{0}^{2} n_{G}^{2}
\end{aligned}
$$

where $\beta=k_{0} N_{\text {eff }}$, with $k_{0}=2 \pi / \lambda_{0}$, and $N_{\text {eff }}$ is the effective index of the waveguide incident mode for $z<0$. After $K_{x}$ and $K_{z}$ are defined, the period and the slant angle of the grating can be determined by the equations

$$
\begin{aligned}
\Lambda & =\frac{2 \pi}{\left(K_{x}^{2}+K_{z}^{2}\right)^{\frac{1}{2}}} \\
\phi & =\tan ^{-1}\left(\frac{K_{z}}{K_{x}}\right) .
\end{aligned}
$$

In practice the angle $\theta_{s}=0 \mathrm{deg}$, which means that the diffracted power is propagating along the $-x$-axis.

Using the leaky-mode approach in conjunction with the rigorous coupled-wave analysis, the radiation coupling coefficient $\alpha$ can be calculated. The waveguide film layer thickness was selected again to be $t_{2}=1.0 \mu \mathrm{m}$. The radiation coupling coefficient is shown as a function of the waveguide film layer and photodetector layer separation $t_{1}$ in Fig. 8(a) and (b) for both $\mathrm{TE}_{0}$ and $\mathrm{TM}_{0}$ incident polarizations and for both $\lambda_{0}=1.3$ and $1.55 \mu \mathrm{m}$ free-space wavelengths. In these results, the effects of both the grating diffraction and the radiation of the waveguide mode due to the presence of the high-index photodetector layer are taken into account. It can be seen that, for $t_{1}<3 \mu \mathrm{m}$, the evanescent coupling dominates the coupling process. However, as was mentioned previously, the evanescent coupling decreases exponentially with the distance $t_{1}$. Therefore, for distances $t_{1}>3 \mu \mathrm{m}$, the coupling process is due solely to the diffraction by the grating. This can be clearly seen in Fig. 8(b), where the radiation coupling coefficient oscillates as a function of $t_{1}$. In this range of $t_{1}$ values, the effect of the evanescent coupling is negligible and the oscillations are due to Fabry-Perot multiple interference effects due to the changing $t_{1}$ distance. It is worth mentioning that the radiation coupling coefficient that

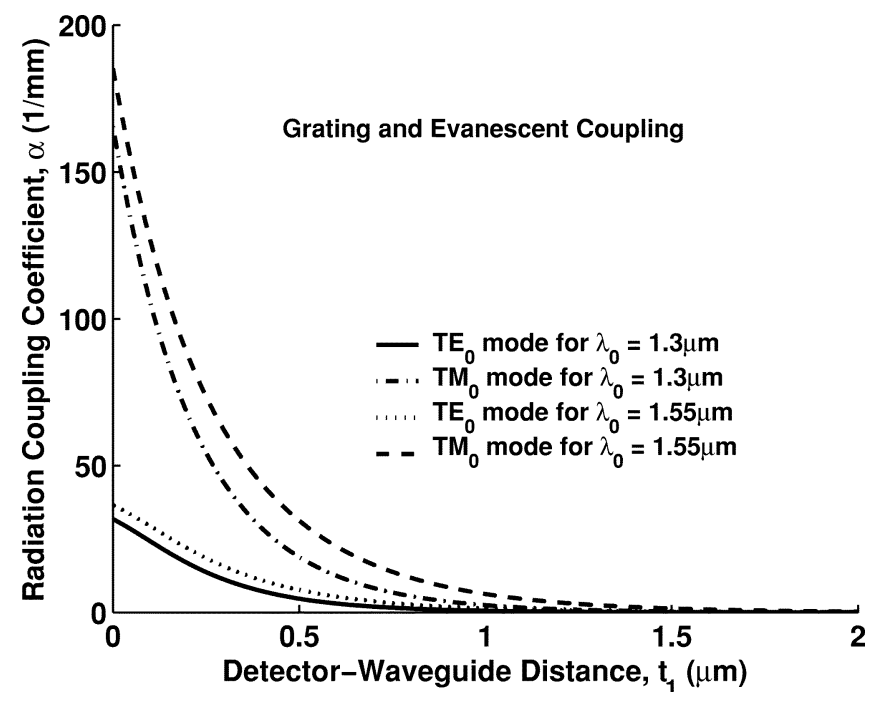

(a)

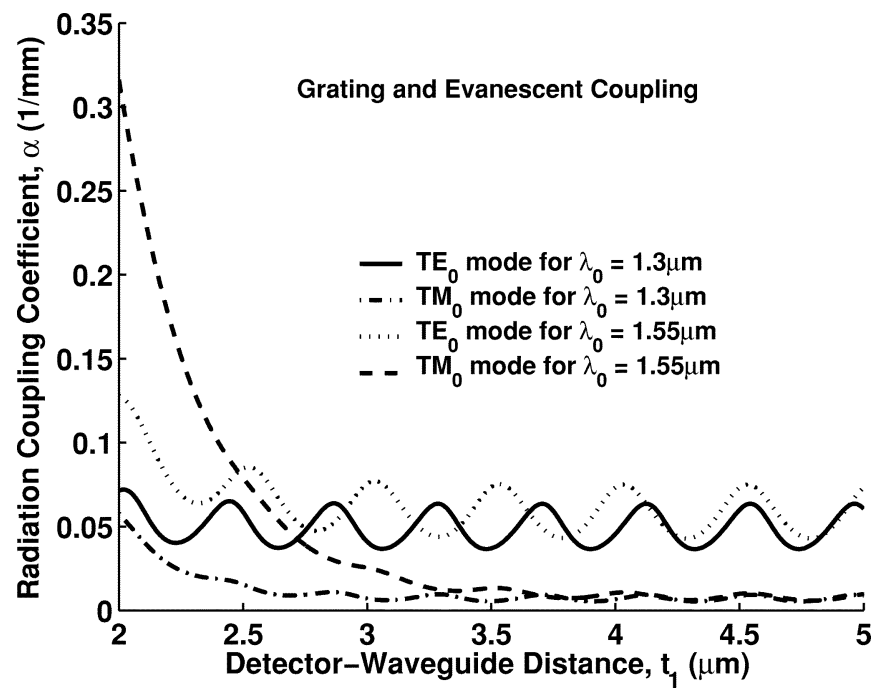

(b)

Fig. 8. Radiation coupling coefficient $\alpha$ for the leaky waveguide formed in the region $z>0$ as a function of detector-waveguide (film) distance $t_{1}$ for the case of the holographic-grating coupler architecture. The various line types show the radiation coupling coefficient $\alpha$ for both $\mathrm{TE}_{0}$ and $\mathrm{TM}_{0}$ incident modes as well as for $\lambda_{0}=1.3$ and $1.55 \mu \mathrm{m}$ free-space wavelengths. (a) The range of $t_{1}=0-2 \mu \mathrm{m}$, where the evanescent coupling dominates. (b) The range of $t_{1}=2-5 \mu \mathrm{m}$, where the holographic-grating coupling dominates.

is due to the grating diffraction alone is almost two to three orders of magnitude smaller than the radiation coupling coefficient that is due to the evanescent coupling alone. Therefore, the coupling of the grating for the parameters listed in this section is very small, making use of the grating inefficient since it would require detector layer lengths in the excess of $L=1000 \mu \mathrm{m}$ to achieve a $P_{\text {det }} / P_{\text {inc }}=P(z=L) / P_{\text {inc }}$ ratio on the order of $5-15 \%$. This is mainly due to the tight confinement of the guided mode in the film region due to the high refractive index of the Ultem layer. If a smaller refractive index medium is selected, the grating can be much more effective, as will be shown in the case of the flip-chip-bonded-photodetector architecture.

Another important observation in the grating coupling architecture is that the radiation coupling coefficient of the TM polarization is much smaller than its TE polarization counterpart. This can be observed in Fig. 8(b). This effect is expected in 
volume holograms and can be explained by the model of Kogelnik [30]. According to this simple two-coupled wave model when the angle between the incident and the diffracted wave is $90^{\circ}$, there is no coupling if the polarization is TM (electric field lying in the plane of incidence). In the waveguide film layer, the mode (incident field) is composed of two plane waves that form an angle (zigzag angle) near $90^{\circ}$ with the boundary normal, thus resulting in nearly $90^{\circ}$ separation between the incident and the diffracted fields (for a designed outcoupled angle $\theta_{s}=0^{\circ}$ ).

\section{Holographic-Grating Coupling From Waveguide to Flip-Chip-Bonded Photodetector}

In the case of the holographic-grating coupling to flip-chipbonded photodetector architecture, the design of the grating is similar to the previously examined case. The grating is designed in such a way that the first-order diffracted toward the photodetector region would be normal or at a specified angle to the layer interfaces. Furthermore, the slanted grating configuration guarantees that a negligible amount of light will be diffracted into the substrate direction. The grating design is based on the phase-matching conditions for the first diffracted order [23], [24]. If the desired order along the $+x$-direction forms an angle $\theta_{c}$ with respect to the $+x$-axis then the grating vector components $K_{x}$ and $K_{z}$ are given by

$$
\begin{aligned}
\beta-K_{z} & =k_{0} n_{c} \sin \theta_{c} \\
\left(\beta-K_{z}\right)^{2}+K_{x}^{2} & =k_{0}^{2} n_{G}^{2}
\end{aligned}
$$

where all parameters are defined as in the previous case. In practice the angle $\theta_{c}=0^{\circ}$, which means that the diffracted power is propagating along the $+x$-axis.

In this case, in order to increase the diffraction efficiency of the grating, a film layer of lower index (BCB of $n_{f}=1.55$ ) was used in conjunction with a lower index substrate material $\left(\mathrm{SiO}_{2}\right.$ of $\left.n_{s}=1.45\right)$. The film layer thickness was selected to be $t_{2}=0.6 \mu \mathrm{m}$ that permits single-mode operation. The lower refractive index as well as the smaller film-layer thickness causes the guided mode to be less confined in the film layer. As a result, the optical field penetrates more into the grating region and consequently is more strongly diffracted. The separation between the photodetector and film layer was selected to be $t_{1}>3.5 \mu \mathrm{m}$, so the effect of the evanescent coupling is negligible. Therefore, the radiation coupling coefficient that is determined using the rigorous coupled-wave analysis in conjunction with the leaky mode approach is due solely to the grating. The total normalized power [as determined by (6)] that is coupled into the flip-chip-bonded photodetector layer $P_{\text {det }} / P_{\text {inc }}=P(z=L) / P_{\text {inc }}$ is shown in Fig. 9 as a function of the detector length $L$ for both $\mathrm{TE}_{0}$ and $\mathrm{TM}_{0}$ incident modes and for both $\lambda_{0}=1.3$ and $1.55 \mu \mathrm{m}$ free-space wavelengths. As was expected for the wave diffracted normal to the boundary, the $\mathrm{TM}_{0}$ case has very small radiation coupling coefficients, resulting in a very small amount of power that can be coupled via the grating. This effect is characteristic of the performance of volume gratings when the angle between the incident and the reflected wave is about $90^{\circ}$, as was discussed in the previous section. The percent of reflected power $R$ was calculated by the FDFD method and was about $3.4 \%$ for TE polarization. The

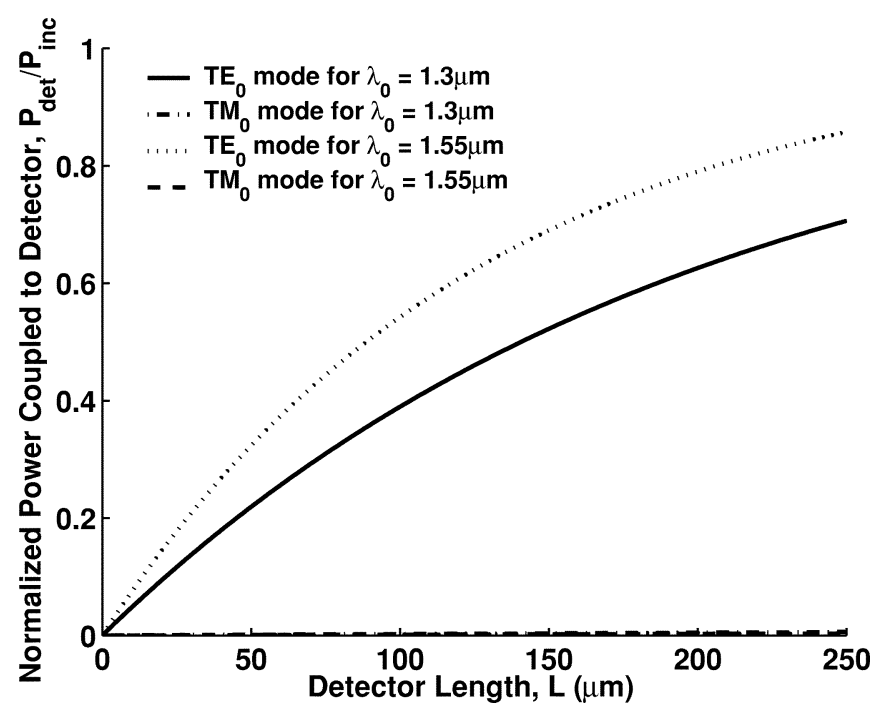

Fig. 9. Normalized power coupled to the detector $P_{\text {det }} / P_{\text {inc }}=P(z=$ $L) / P_{\text {inc }}$ for the leaky waveguide formed in the region $z>0$ as a function of the detector length for the case of the holographic-grating coupling with the flip-chip-bonded photodetector architecture. The various line types show the normalized power $P_{\text {det }} / P_{\text {inc }}$ for both $\mathrm{TE}_{0}$ and $\mathrm{TM}_{0}$ incident modes for $\lambda_{0}=1.3$ and $1.55 \mu \mathrm{m}$.

preferential coupling efficiencies $\eta_{D}$ were $98.99 \%$ and $99.89 \%$ for $\lambda_{0}=1.3$ and $1.55 \mu \mathrm{m}$ free-space wavelengths for the TE polarization. The corresponding parameters for TM polarization were less than $50 \%$, but this was due to the very inefficient diffraction process in the TM cases. For detector lengths up to $250 \mu \mathrm{m}$, the percentage of the power that is coupled into the photodetector can be about $70-85 \%$.

\section{FABRICATION ISSUES}

One optical interconnection integration approach is to use embedded emitters/photodetectors, which have the optical signals originate and/or terminate in the waveguide directly on the board, without optical beam turning. Optical interconnections with integrated waveguides and optoelectronic $(\mathrm{OE})$ devices in the substrate and epilayers have been reported in compound semiconductors [8], [9], [31]-[34], such as InP-based materials, with reported high coupling efficiency and monolithic integration. Polymer waveguides integrated onto Si [33] or GaAs [34], [35] electrical interconnection substrates that have photodetectors fabricated in the substrate, thus creating embedded waveguide interconnections, have been demonstrated. However, this approach does not accommodate noncompound semiconductor substrates, such as organic substrates. An embedded waveguide approach uses thin-film $\mathrm{OE}$ devices (with the $\mathrm{OE}$ device growth substrate removed), which can be bonded to any host substrate, including organic materials. The polymer waveguide material can then be deposited directly onto the thin-film active OE devices, which are thus embedded directly into the waveguide core, or embedded in the cladding.

This embedded optoelectronic waveguide interconnection technology for OE devices creates an optical interconnection option on the board/module/chip, which has exclusively electrical inputs and/or outputs (but can have optical inputs/outputs as well, if desired for line card to backplane perpendicular 
interconnections, for example). Optical inputs that can be implemented for such planar interconnects include a coupled fiber, or embedded thin-film edge-emitting lasers (at wavelengths including $850,980,1300$, and $1550 \mathrm{~nm}$ ) that emit directly into the waveguide structure, for a source that originates on the board. Implementing these types of planar lightwave circuit (PLC) optical interconnections with embedded emitters and detectors may eliminate the need for optical beam-turning elements that route the beam perpendicular to the surface of the board/module/chip, and reduces waveguide to active $\mathrm{OE}$ device optical alignment to an OE device assembly step with sequentially aligned masking steps, which mirrors integrated circuit fabrication. In the future, the integration of additional PLC passive and further active embedded devices creates multiplexing and optical signal-processing options for more complex integrated optical/electrical microsystems, enabling the designer to choose functions from optical and electrical integrated "toolboxes" that offer the best of both integrated sets of functionality. The electrical interface circuits can be connected directly to the embedded active OE devices, through either wire bonding or bump bonding (as rising data rates preclude wire bonding), or through the electrical interconnection lines on the substrate. The assembly tradeoff that is inherent in the embedded optical waveguide interconnection is that the $\mathrm{OE}$ active devices are bonded directly to the substrate rather than bumped to the substrate. To minimize the impact of introducing optical interconnections into electrical interconnection substrates, the embedded $\mathrm{OE}$ waveguide interconnections can be integrated onto a fabricated electrical interconnection substrate through postprocessing. To enhance yield, at the board/module level, the optical interconnections can be electrically tested before the integrated circuits are assembled onto the board.

Independently optimized waveguides, embedded thin-film photodetectors, and a standard Si substrate (which can be used as an electrical interconnection substrate) have been integrated and tested [36]. Fig. 10(a) and (b) contains a schematic and a photomicrograph of a thin-film InGaAs-based inverted MSM photodetector bonded to pads with subsequent waveguide integration on top of the photodetector. Integration processes and measurement results are presented in the next section. Specifically, the integrated structure consists of a thin-film InP-based photodetector in a configuration of an Ultem (core)/BCB (clad) polymer waveguide integrated onto a Si interconnection substrate, with the photodetector integrated into the BCB cladding. The use of thin-film photodetectors (with the $\mathrm{OE}$ device growth substrate removed) enables the photodetectors to be bonded and electrically connected to the $\mathrm{Si}$ interconnection substrate and embedded in the polymer optical waveguides. Because the photodetector is embedded in substrate layer, the optical signal can be coupled from the waveguide into the photodetector without the use of beam turning elements. Using this heterogeneous integration technology, the thin-film $\mathrm{OE}$ devices, optical waveguides, and electrical interconnection media can be optimized separately for optimal mixed optical and electrical signal distribution for board-, module-, and chip-level interconnections.

In the cases where volume holographic gratings are needed, the holographic recording material used in this research is the DuPont OmniDex613 (HRF600X). This photosensitive

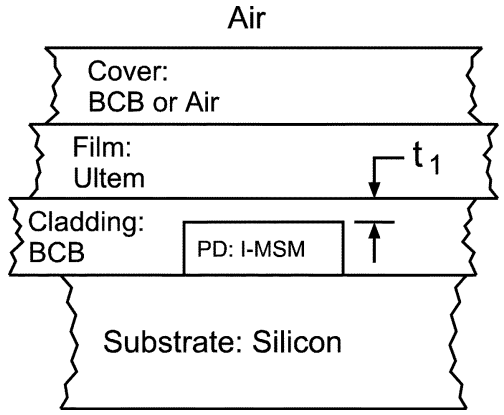

(a)

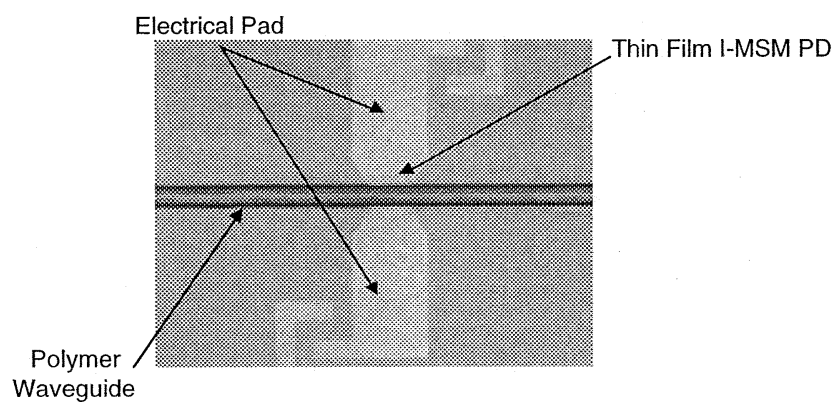

(b)

Fig. 10. (a) Cross-sectional view of the fabricated and tested samples (b) Photomicrograph of a thin-film InP-based inverted-MSM photodetector bonded to electrical connection pads on $\mathrm{S} \mathrm{SiO}_{2} / \mathrm{Si}$ substrate. The photodetector is embedded in a BCB organic polymer waveguide structure, which was deposited onto the substrate/photodetector.

polymer is coated from a solution in an organic solvent onto a 50- $\mu \mathrm{m}$-thick Mylar base with a removable 25- $\mu \mathrm{m}$ Mylar cover sheet. The film in which the grating on the waveguide can be recorded [as shown in Fig. 1(b) and (c)] is accomplished by means of cutting a piece out of a large sheet, removing the cover sheet, exposing it with the required interference pattern, and laminating it onto the desired surface. To record the slanted grating, high-index prisms should be used in order to achieve the required interference pattern within the photopolymer film layer. A two-beam interferometric configuration is used for the exposure in conjunction with an Ar-ion ultraviolet wavelength of $363.8 \mathrm{~nm}$. The optimal recording conditions to obtain high diffraction efficiency grating couplers can be determined [37], [38]; they depend on the exposure intensity, exposure dosage, and postbaking after exposure. After the grating is recorded, it can be laminated onto the waveguide; then the Mylar layer can be removed. This summarizes the procedure for obtaining the gratings shown in architectures of Fig. 1(b) and (c).

\section{COMPARISON OF THEORY AND EXPERIMENT}

In this section, the presented analysis and experimental results are compared in the case of evanescent coupling from waveguide to substrate-embedded photodetector. Two samples have been fabricated and tested. The sample configurations fall under the general structure shown in Fig. 1(a). A more detailed configuration of the fabricated devices is shown in Fig. 10(a), and a microphotograph is shown in Fig. 10(b). Sample no. 1 is composed of an air cover, an overcladding BCB layer of thickness of $2.0 \mu \mathrm{m}$, an Ultem film layer of thickness of $1.0 \mu \mathrm{m}$, a $\mathrm{BCB}$ under cladding of thickness $\sim 1 \mu \mathrm{m}$ (on top of the 
TABLE II

Comparison of Theoretical Coupling EfFICIENCIES $P_{\text {det }} / P_{\text {inc }}$ TO EXPERIMENTALLY OBTAINED ONES FOR THE CASE OF THE EVANESCENT Coupling From Waveguide to Substrate-Embedded Photodetector

\begin{tabular}{ccccc}
\hline \hline \multicolumn{5}{c}{ Sample No. 1 } \\
\hline \hline Distance $t_{1}$ & \multicolumn{5}{c}{ Analytical $P_{\text {det }} / P_{\text {inc }}$} & Measured $P_{\text {det }} / P_{\text {inc }}$ \\
$(\mu \mathrm{m})$ & TE Pol. (\%) & TM Pol. $(\%)$ & Avg. (\%) & $(\%)$ \\
\hline 0.8 & 6.33 & 22.68 & 14.51 & 11.83 \\
1.0 & 2.73 & 10.70 & 6.72 & - \\
1.2 & 1.18 & 4.87 & 3.03 & - \\
\hline \hline \multicolumn{5}{c}{ Sample No. 2 } \\
\hline \hline Distance $t_{1}$ & Analytical $P_{\text {det }} / P_{\text {inc }}$ & Measured $P_{\text {det }} / P_{\text {inc }}$ \\
$(\mu \mathrm{m})$ & TE Pol. (\%) & TM Pol. (\%) & Avg. $(\%)$ & $(\%)$ \\
\hline 0.2 & 30.01 & 65.97 & 47.99 & - \\
0.4 & 14.63 & 42.47 & 28.55 & - \\
0.6 & 6.10 & 21.09 & 13.60 & 9.39 \\
\hline \hline
\end{tabular}

photodetector), and the embedded in the undercladding I-MSM InP-based photodetector. The structure is on top of a silicon microelectronic substrate. Sample no. 2 is composed of an air cover (overcladding), an Ultem film layer of thickness of $1.8 \mu \mathrm{m}$, and a BCB under cladding of thickness $\sim 0.2 \mu \mathrm{m}$ (on top of the photodetector). Again the I-MSM InP-based photodetector is embedded in the BCB under cladding. As in the first sample, the structure has been grown on top of a silicon microelectronics substrate. Details about the fabrication process can be found in [36]. For both samples, the testing was done using a laser diode of $1.3 \mu \mathrm{m}$ free-space wavelength coupled into a multimode fiber. Also for both samples, the photodetector length was $L=150 \mu \mathrm{m}$. Light from the end of the fiber was focused on the polished edge of the film layer of the waveguide structure for both samples, and sufficient coupling was achieved. The dark current and the responsivity of the I-MSM photodetector were measured before and after the waveguide fabrication. The dark current varied depending on the applied voltage between $0.1-50$ $\mathrm{nA}$. The responsivity was measured to be $0.4 \mathrm{~A} / \mathrm{W}$ at $5 \mathrm{~V}$. More details about these can be found in [36].

The experimental coupling efficiency of the light that was evanescently coupled from the waveguide into the embedded photodetector was estimated by measuring the photocurrent and knowing the responsivity of the photodetector. The amount of input power was estimated theoretically using a finite-difference beam-propagation method. Therefore, the experimentally obtained values of the coupling efficiencies are shown in Table II (as measured $P_{\text {det }} / P_{\text {inc }}$ ) for both samples. Variation in the photodetector and the BCB under cladding thicknesses make the actual knowledge of the separation $t_{1}$ between the photodetector and the film waveguide layer [see Figs. (1a) and (10a)] uncertain within fractions of a micrometer. The coupling efficiency $P_{\text {det }} / P_{\text {inc }}$ depends strongly on the separation distance $t_{1}$, as can be seen in Fig. 5(a) and (b). Therefore, in order to compare the present analysis with the experimentally obtained results, the separation $t_{1}$ was varied by a few tenths of a micrometer to accommodate the uncertainty of the actual separation $t_{1}$. Both the theoretical and experimental results are shown in Table II. During the experiment, it was known that the input light was not polarized. Therefore, in Table II, both the TE and TM polarization results are shown, as well as their average (assuming equal amount of TE and TM light). From the theoretical results, it can be observed that the actual waveguide-film/photodetector separation $t_{1}$ was near 0.8 and $0.6 \mu \mathrm{m}$ for samples 1 and 2 , respectively, which is well within the range of uncertainty of $t_{1}$. The experimental measurements were slightly lower than the theoretical results since the scattering losses were not considered in the analysis.

\section{SUMMARY AND CONCLUSIONS}

In this paper, potentially low-cost fully substrate-embedded or flip-chip-bonded optical interconnect architectures have been analyzed and compared. The architectures involve a volume-holographic grating coupling for beam turning and/or an embedded thin-film inverted-MSM photodetector for evanescent coupling. The architectures can be integrated onto silicon microelectronic substrates with InP-material-based thin-film photodetectors and optical quality polymer layers that can be used as waveguides and/or volume holographic gratings. Similar architectures can be implemented on low-cost organic substrates such as FR4 epoxy without any significant theoretical modifications. Three architectures have been investigated as follows: a) an evanescent-coupling architecture with a substrate-embedded photodetector, b) a volume-holographic-grating coupler architecture with a substrate-embedded photodetector, and c) a volume-holographic-grating coupler architecture with a flip-chip-bonded photodetector.

It has been found that the evanescent-coupling architecture can be very efficient for both polarizations (TE and TM) provided that the distance between the waveguide film layer and the photodetector layer $\left[t_{1}\right.$ in Fig. 1(a)] is small compared to the free-space wavelength. In this case, power from the waveguide is radiated and absorbed into the photodetector. However, as distance $t_{1}$ increases, the radiation coupling coefficient decreases exponentially. Furthermore, the evanescent-coupling architecture is not in general very wavelength-sensitive. On the other hand, the architectures that involve a volume holographic grating can be useful either when the distance $t_{1}$ is large or when there is a need for wavelength/polarization sensitivity [in the case of the architecture with a substrate-embedded photodetector shown in Fig. 1(b)]. In the case of the architecture with the flip-chip-bonded photodetector [Fig. 1(c)], the grating is absolutely necessary since the separation between the waveguide and the photodetector is large and no evanescent coupling can occur. In the latter case, slanted volume gratings offer the advantages of alignment insensitivity as well as preferential coupling. However, for normal outcoupling, the volume holographic grating is efficient in the case of TE polarization but very inefficient in the case of TM polarization. If the desired direction of the outcoupled power is about $45^{\circ}$ instead of normal to the interfaces, then both TE and TM polarizations can become equally efficient [24]. Furthermore, the grating coupling architectures can become more efficient if the waveguide/grating combination is designed in such a way that the optical mode extends well into the grating region, thus enhancing the interaction between the guided mode and the grating.

In addition, some of the fabrication issues for low-cost implementations of the abovementioned architectures have 
been discussed. Architectures involving evanescent coupling with substrate-embedded photodetectors have already been implemented [36], while architectures involving volume holographic gratings are currently under investigation.

\section{REFERENCES}

[1] E. D. Kyriakis-Bitzaros, N. Haralabidis, M. Lagadas, A. Georgakilas, Y. Moisiadis, and G. Halkias, "Realistic end-to-end simulation of the optoelectronic links and comparison with the electrical interconnections for system-on-chip applications," J. Lightwave Technol., vol. 19, pp. 1532-1542, Oct. 2001.

[2] W. Ryu, J. Lee, H. Kim, S. Ahn, N. Kim, B. Choi, D. Kam, and J. Kim, "RF interconnect for multi-Gbit/s board-level clock distribution," IEEE Trans. Adv. Packag., vol. 23, pp. 398-407, Aug. 2000.

[3] D. A. B. Miller, "Rationale and challenges for optical interconnects to electronic chips," Proc. IEEE, vol. 88, pp. 728-749, June 2000.

[4] S. K. Tewksbury and L. A. Hornak, "Optical clock distribution in electronic systems," J. VLSI Signal Process. S, vol. 16, pp. 225-246, June-July 1997.

[5] M. Rassaian and M. W. Beranek, "Quantitative characterization of 96.5Sn3.5Ag and 80Au20Sn optical fiber solder bond joints on silicon micro-optical bench substrates," IEEE Trans. Adv. Packag., vol. 22, pp. 86-93, Feb. 1999.

[6] S. J. Walker and J. Jahns, "Optical clock distribution using integrated free-space optics," Opt. Commun., vol. 90, pp. 359-371, June 15, 1992

[7] P. J. Delfyett, D. H. Hartman, and S. Z. Ahmad, "Optical clock distribution using a mode-locked semiconductor laser-diode system," J. Lightwave Technol., vol. 9, pp. 1646-1649, Dec. 1991.

[8] Y. Liu, L. Lin, C. Choi, B. Bihari, and R. T. Chen, "Optoelectronic integration of polymer waveguide array and metal-semiconductor-metal photodetector through micromirror couplers," IEEE Photon. Technol. Lett., vol. 13, pp. 355-357, Apr. 2001.

[9] R. T. Chen, L. Lin, C. C. Choi, Y. J. Liu, B. Bihari, L. Wu, S. Tang, R. Wickman, B. Picor, M. K. Hibbs-Brenner, J. Bristow, and Y S. Liu, "Fully embedded board-level guided-wave optoelectronic interconnects," Proc. IEEE, vol. 88, pp. 780-793, June 2000.

[10] A. W. Snyder and J. D. Love, Optical Waveguide Theory. New York: Chapman and Hall, 1996, ch. 24-26.

[11] T. Tamir and A. A. Oliner, "Guided complex waves. Part 2: relation to radiation patterns," Proc. Inst. Elect. Eng., vol. 110, pp. 325-334, Feb. 1963.

[12] E. Anemogiannis and E. N. Glytsis, "Multilayer waveguides: efficient numerical analysis of general structures," J. Lightwave Technol., vol. 10, pp. 1344-1351, Oct. 1992.

[13] R. E. Smith, S. N. Houde-Walter, and G. W. Forbes, "Numerical determination of planar waveguide modes using the analyticity of the dispersion relation," Opt. Lett., vol. 16, pp. 1316-1318, Sept. 1, 1991.

[14] L. M. Delves and J. N. Lyness, "A numerical method for locating the zeros of an analytic function," Math. Comp., vol. 21, pp. 543-560, 1967.

[15] M. N. O. Sadiku, Numerical Techniques in Electromagnetics. Boca Raton, FL: CRC Press, 1992, ch. 3.

[16] W.-C. Liu and M. W. Kowarz, "Vector diffraction from subwavelength optical disk structures: two-dimensional modeling of near-field profiles, far-field intensities, and detector signals from DVD," Appl. Opt., vol. 38, pp. 3787-3797, Oct. 10, 1999.

[17] S.-D. Wu and E. N. Glytsis, "Finite-number-of-periods holographic gratings with finite-width incident beams: analysis using the finite-difference frequency-domain method," J. Opt. Soc. Amer. A, vol. 19, pp. 2018-2029, Oct. 2002.

[18] J. P. Bérenger, "Improved PML for the FDTD solution of wave-structure interaction problems," IEEE Trans. Antennas Propagat., vol. 45, pp. 466-473, Mar. 1997.

[19] S. T. Peng, T. Tamir, and H. L. Bertoni, "Leaky-wave analysis of optical periodic couplers," Electron. Lett., vol. 9, pp. 150-152, Mar. 22, 1973.

[20] — , "Theory of periodic dielectric waveguides," IEEE Trans. Microwave Theory Tech., vol. MTT-23, pp. 123-133, Jan. 1975.

[21] W. Driemeier, "Coupled-wave analysis of the Bragg effect waveguide coupler," J. Mod. Opt., vol. 38, pp. 363-377, Feb. 1991.

[22] S. M. Schultz, E. N. Glytsis, and T. K. Gaylord, "Volume grating preferential-order focusing waveguide coupler," Opt. Lett., vol. 24, pp. 1707-1710, Dec. 1, 1999.

[23] — , "Design, fabrication, and performance of preferential-order volume grating waveguide couplers," Appl. Opt., vol. 39, pp. 1223-1231, Mar. 10, 2000
[24] R. A. Villalaz, E. N. Glytsis, and T. K. Gaylord, "Volume grating couplers: polarization and loss effects," Appl. Opt., vol. 41, pp. 5223-5229, Sept. 1, 2002.

[25] M. G. Moharam and T. K. Gaylord, "Rigorous coupled-wave analysis of planar-grating diffraction,” J. Opt. Soc. Amer., vol. 71, pp. 811-818, July 1981.

[26] M. G. Moharam, D. A. Pommet, E. B. Grann, and T. K. Gaylord, "Stable implementation of the rigorous coupled-wave analysis of surface-relief gratings: enhanced transmittance matrix approach," J. Opt. Soc. Amer. A, vol. 12, pp. 1077-1086, May 1995.

[27] M. Nevière, "The homogeneous problem," in Electromagnetic Theory of Gratings, R. Petit, Ed. New York: Springer-Verlag, 1980, ch. 5.

[28] M. Pecht and X. Wu, "Characterization of polyimides used in high density interconnects," IEEE Trans. Comp. Packag. B, vol. 17, pp. 632-639, Nov. 1994.

[29] R. A. Kirchhoff, C. J. Carriere, K. J. Bruza, N. G. Rondan, and R. L. Sammler, "Benzocyclobutenes: a new class of high performance polymers," J. Macromol. Sci. Chem. A, vol. 28, pp. 1079-1113, 1991.

[30] H. Kogelnik, "Coupled wave theory for thick hologram cases," Bell Syst. Tech. J., vol. 48, pp. 2909-2947, Nov. 1969.

[31] S. Kollakowski, A. Strittmatter, E. Dröge, E. H. Böttcher, D. B. O. Reimann, and K. Janiak, "65 GHz InGaAs/InAlGaAs/InP waveguide-integrated photodetectors for the 1.3-1.55 $\mu \mathrm{m}$ wavelength regime," Appl. Phys. Lett., vol. 74, pp. 612-614, Jan. 25, 1999.

[32] E. H. Böttcher, H. Pfitzenmaier, E. Dröge, S. Kollakowski, A Stittmatter, D. Bimberg, and R. Steingrüber, "Distributed waveguide-integrated InGaAs MSM photodetectors for high-efficiency and ultra-wideband operation," in Proc. Conf. 11th Conf. InP and Related Material (IPRM99), May 16-20, 1999, pp. 79-82.

[33] C. H. Buchal, A. Roelofs, M. Siegert, M. Löken, K. Nashimoto, R. Pachter, B. W. Wessels, J. Shmulovich, A. K.-Y. Jen, K. Lewis, R. Sutherland, and J. W. Perry, "Polymeric strip waveguides and their connection to very thin ultrafast metal-semiconductor-metal detectors," in Proc. Conf. Thin Films for Optical Waveguide Devices and Materials for Optical Limiting (Materials Research Society Symp. Proc. Vol. 597), Nov. 30-Dec. 3 1999, pp. 97-102.

[34] F. Gouin, L. Robitaille, C. L. Callender, J. Noad, and C. Almeida, "A $4 \times$ 4 optoelectronic switch matrix integrating an MSM array with polyimide optical waveguides," in Proc. SPIE, vol. 3920, 1997, pp. 287-295.

[35] C. L. Callender, L. Robitaille, J. P. Noad, F. Gouin, and C. Almeida, "Optimization of metal-semiconductor-metal (MSM) photodetector arrays integrated with polyimide waveguides," in Proc. SPIE, vol. 2918, 1997, pp. 211-221.

[36] S.-Y. Cho, M. A. Brooke, and N. M. Jokerst, "Optical interconnections for electrical boards using embedded active optoelectronic components," IEEE J. Select. Topics Quantum Electron., vol. 9, Mar./Apr. 2004, to be published.

[37] S. M. Schultz, "High efficiency volume grating couplers," Ph.D. dissertation, Georgia Inst. of Technology, 1999.

[38] S.-D. Wu and E. N. Glytsis, "Holographic grating formation in photopolymers: parameter determination based on a nonlocal diffusion model and the rigorous coupled-wave analysis," J. Opt. Soc. Amer. B, vol. 20, pp. 1177-1188, June 2003.

Elias N. Glytsis (S'81-M'81-SM'91) received the Ph.D. degree from the Georgia Institute of Technology, Atlanta, in 1987.

He joined the Faculty of the School of Electrical and Computer Engineering, Georgia Institute of Technology, as an Assistant Professor in January 1988 and has been a Professor since 2000. His current research interests are in electromagnetic theory of diffractive optical elements, photonic bandgap diffractive elements and devices, optical interconnections for optoelectronic packaging, long-period fiber gratings, optoelectronic devices, semiconductor quantum devices such as intersubband emitters and detectors, and design/optimization/integration software. He has published more than 95 journal publications and 80 conference papers. He has received eight U.S. patents. He has been a co-Guest Editor of two special issues of the Optical Society of America on grating diffraction. He has been a Topical Editor of the Journal of Optical Society of America $A$ on scattering and grating diffraction 1992-1997. He has also been a Guest Editor of the Microelectronics Journal of the October 1999 special issue on quasi-bound states in quantum heterostructure devices.

Dr. Glytsis is a Fellow of the Optical Society of America (OSA) and Member of the IEEE Lasers and Electro-Optic Society (LEOS) and the Greek Society of Professional Engineers. 
Nan M. Jokerst (S'83-M'88-SM'98-F'03) received the Ph.D. degree from the University of Southern California, Los Angeles, in 1989.

She is the Joseph M. Pettit Professor of Optoelectronics in the School of Electrical and Computer Engineering, Georgia Institute of Technology (Georgia Tech), Atlanta. She joined the Electrical Engineering Faculty, Georgia Tech, in 1989. She is the Optoelectronics Thrust Leader for the Georgia Tech National Science Foundation Engineering Research Center in Electronic Packaging. She has published and presented more than 175 papers and three book chapters. She has received three patents and has two pending. She has organized and served on numerous conference committees. She was on the Board of Directors of the Optical Society of America as Chair of the Engineering Council.

Dr. Jokerst received the IEEE Millennium Medal in 2000. She is a Fellow of the Optical Society of America (OSA). She received the Harriet B. Rigas Education Award from the IEEE Education Society, a DuPont Young Faculty Award, a National Science Foundation Presidential Young Investigator Award, a Newport Research Award, and three teaching awards. She was a Hewlett-Packard Fellow. She was an elected Member the IEEE Lasers and Electro-Optic Society (LEOS) Board of Governors and Vice President of Conferences for IEEE LEOS. She has also served as elected Chair, Vice Chair, Secretary, and Treasurer of the Atlanta IEEE Section.

Ricardo A. Villalaz (S'97) received the B.Eng. degree and the M.S. degree in electrical and computer engineering from the Georgia institute of Technology, Atlanta, in 1998 and 2000, respectively, where he is currently pursuing the Ph.D. degree.

His current research interests are in volume grating couplers for optical interconnections.

Mr. Villalaz is a Student Member of the Optical Society of America (OSA) and the Society of Hispanic Professional Engineers, and a Member of Tau Beta $\mathrm{Pi}$ and Eta Kappa Nu.

Sang-Yeon Cho (S'00) received the B.S. and M.S. degrees in electrical and computer engineering from SungKyunKwan University, Korea, in 1997 and 1998, respectively. He received the M.S.E.C.E. degree from the Georgia Institute of Technology, Atlanta, in 2000, where he is currently pursuing the Ph.D. degree.

His research interest is in high-speed optical interconnection using thin-film active OE devices with polymer optical waveguides.

Shun-Der Wu (S'00) was born in Tainan, Taiwan, R.O.C., in 1973. He received the B.S. and M.S. degrees from the Department of Engineering Science, National Cheng-Kung University, Tainan, in 1995 and 1997, respectively. He is currently pursuing the $\mathrm{Ph} . \mathrm{D}$. degree in electrical engineering at the Georgia Institute of Technology, Atlanta.

His current research interests include numerical analysis of diffractive optical elements and optical interconnections for packaging systems.

Mr. Wu is a Student Member of the Optical Society of America (OSA).
Zhaoran Huang (S'98) received the B.S. degree from the Beijing Institute of Technology, China, in 1995 and the M.S. degree in electrical and computer engineering from the Georgia Institute of Technology, Atlanta, in 1999, where she is currently pursuing the $\mathrm{Ph} . \mathrm{D}$. degree.

Her research interests include multigigahertz InP/InGaAs MSM photodetectors in applications of optical waveguide interconnections and photoreceivers.

Martin A. Brooke (S'85-M'86) received the B.Elect. degree (first-class honors) from Auckland University, New Zealand, in 1981 and the M.S. and $\mathrm{Ph} . \mathrm{D}$. degrees in electrical engineering from The University of Southern California, Los Angeles, in 1984 and 1988, respectively.

He is currently an Associate Professor of electrical engineering at the Georgia Institute of Technology, Atlanta. He has received four U.S. patents. He has published more than 100 articles in technical journals and proceedings. Articles on his work have appeared in several trade publications. His expertise is in high-speed high-performance signal processing. His current projects include learning neural network hardware development; neural network prediction of turbulent flow; focal plane image processing hardware development; $1-20 \mathrm{Gbps}$ digital CMOS transceiver circuits for low-cost fiber-optic communication; nonlinear filtering algorithms for telecommunications; nonlinear analog-to-digital converter design; accurate modeling of high-speed circuit parasitics; and statistically relevant device models for accurate prediction of high performance integrated circuit yield.

Prof. Brooke won a National Science Foundation Research Initiation Award in 1990 and the 1992 IEEE Midwest Symposium on Circuits and Systems Myril B. Reed Best Paper Award.

Thomas K. Gaylord (S'65-M'70-SM'77-F'83) received the B.S. degree in physics and the M.S. degree from the University of Missouri-Rolla. He received the Ph.D. degree in electrical engineering from Rice University, Houston, TX.

He is with the Georgia Institute of Technology, Atlanta, where he is Julius Brown Chair and Regents' Professor of Electrical and Computer Engineering. $\mathrm{He}$ is the author of 350 technical publications and 25 patents in the areas of diffractive optics, optoelectronics, and semiconductor devices.

Dr. Gaylord is a Fellow of the Optical Society of America (OSA) and the American Association for the Advancement of Science. He received the Curtis W. McGraw Research Award from the American Society for Engineering Education; the IEEE Centennial Medal; the IEEE Graduate Teaching Award; the Georgia Tech Outstanding Teacher Award; and the Engineer of the Year Award from the Georgia Society of Professional Engineers. 